\title{
Work and home location: Possible role of social networks
}

\author{
Nebiyou Tilahun ${ }^{\mathrm{a}, *}$, David Levinson ${ }^{\mathrm{b}}$

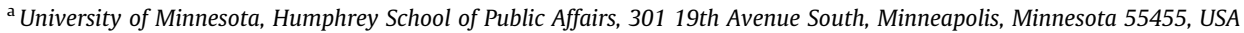 \\ ${ }^{\mathrm{b}}$ University of Minnesota, Department of Civil Engineering, 500 Pillsbury Drive SE, Minneapolis, MN 55455, USA
}

\section{A R T I C L E I N F O}

\section{Article history:}

Received 12 August 2008

Received in revised form 8 May 2010

Accepted 17 July 2010

\section{Keywords:}

Contacts

Job finding

Home finding

Work commute

Social networks

Home and work location sharing

\begin{abstract}
A B S T R A C T
This research explores to what extent people's work locations are similar to that of those who live around them. Using the Longitudinal Employer-Household Dynamics data set and the 2000 decennial census, we investigate the home and work locations of different census block residents in the Twin Cities (Minneapolis-St. Paul) metropolitan area. Our aim is to investigate if people who share a residence neighborhood also share work locations to a degree beyond what would be explained by distance and opportunities around them. Using quadratic assignment procedure we find a significantly higher joint home and work location choice among residents in eight areas selected for this investigation. Further, using data for the entire metropolitan area, we show what socio-demographic variables are associated with higher levels of home-work co-location. We hypothesize that a possible reason for the observed patterns is the role neighborhood level and work place social networks play in locating jobs and residences respectively.
\end{abstract}

(c) 2011 Elsevier Ltd. All rights reserved.

\section{Introduction}

Humans are social animals. We interact with one another, form different types of relationships, maintain ties with one another over time and space. We are continuously collecting information about each other, what we think of particular people, places, goods, etc. A simple question such as "What did you do yesterday?" can garner a range of responses about neighborhood locations, schools, restaurants, and so on. To what extent these interactions inform our understanding of our environment depends on whom the information comes from, the type of relationship we have with them, how convincing they were, and whether the information gets reinforced through other channels. This process along with self experience helps individuals gather, discard, reinforce and refine their perceptions and knowledge about their environment.

On a day to day basis, much of the information gathering is passive. However, when the need arises for a particular good or information, the take-off point for the search is likely to be these stored information that have been built up over time. During an active search, the searcher can return to the original source for in depth answers or may seek alternate sources to refine the search. For example, a person who has mentioned his experience with a particular school district can be asked in depth questions, or one who seems to be well connected to employers of interest in past discussions can be asked to pass on a recommendation. Alternatively the searcher can seek out similar information from other sources in taking future steps.

The roles that social networks and their structure play in a variety of individual and social activities has been a matter of much research. These studies span multiple fields including labor market studies (e.g. Granovetter, 1974; Lindsay and Greig, 2005; Stoessel, 2002; Fontaine, 2008), crime and unemployment (e.g. Calvó-Armengol and Zenou, 2003), health (e.g. Fleury, 1993; Cattell et al., 2001; Pescosolido and Levy, 2002), child care (e.g. Powell, 1979; Hill, 1991), inter-firm alliances (e.g. BarNir and Smith, 2002) and others. More recently the role of social networks in transportation decisions has also received

\footnotetext{
* Corresponding author. Tel.: +1 9524868786 .

E-mail addresses: tila0006@umn.edu (N. Tilahun), dlevinson@umn.edu (D. Levinson).

URL: http://nexus.umn.edu (D. Levinson).
} 
increasing attention. Investigating the role of social networks in social-activity travel, Carrasco et al. (2006) find systematic individual and social network effects that affect the spatial distribution of locations for social interaction. Arentze and Timmermans (2008) propose a framework for incorporating social networks into activity travel-behavior microsimulation. Hackney and Axhausen (2006) propose an agent model of the interdependence of social networks and travel.

In this study we explore whether interaction among social contacts is a significant source of information on jobs and housing. Specifically we are interested in the role neighborhood level and workplace level networks play in work and home finding. Our purpose in focusing on the neighborhood and workplace contacts is driven in part by the implications it has in transportation planning applications.

The neighborhood and workplace are seen as spaces that bring people together and facilitate information sharing. In this sense our conception is similar to that of Feld (1981) who points out that many social networks develop around a focus. The "focus" in his theory can be anything around which joint activities take place including places, people, work, etc. One of his basic propositions maintains that two individuals that are tied to the same focus are more likely to be tied to one another than two people not so related. Each individual can have many foci and different networks arising from their involvement in each, and can play different roles both within the focus and across foci. We will look at the workplace and the residential neighborhood as the foci around which social networks can develop and where information is passed among residents about work and among workers about residence locations.

One can see how sharing a home or work location can lead to higher chances of influence. Two persons, A and B, are more likely to know one another if they work at the same location than two individuals randomly selected in the population. If $A$ and B know one another and have further similarities in other dimensions, then it becomes more likely that they would share their experiences and the possibility for mutual influence grows. On the home neighborhood side as well people who live close to one another have higher chances of knowing one another, and are more likely to interact with and influence one another as compared to two people randomly selected in the population. The possibility for acquaintance and influence increases further if they are involved in similar other activities (for example a home owners association, membership in a neighborhood club or if they have children going to the same school).

The link between where people live and go to work has been a topic of much research in transportation planning. Trip distribution models try to match origins and destinations, often using aggregate zonal variables to estimate the level of interaction between zones by the number of travelers going from an origin zone to a destination zone for different purposes. The most well known and widely used of trip distribution models, the gravity model (Voorhees, 1955), has gone through several evolutionary changes (Easa, 1993,). At its basic, the gravity model distributes trips from origins to destinations in direct proportion to the "size" (or some measure of opportunity) of the origin and destination and inverse proportion to the separation/cost between them. Explicit consideration of the job-finding or home-finding process within these aggregate models has not been made.

In the transportation context, the social network paradigm that is studied in this paper posits that home-work location sharing (co-location) would be higher than what can be explained by travel time, neighborhood characteristics and demographic preferences. If the influence of contacts is significantly observed, one would expect that certain residential neighborhoods produce more workers for a given employment district than can be explained by travel time, and opportunities at the destination alone.

There are of course other mechanisms in which peoples' home and work locations can coincide. Some of the selection criteria that individuals use in selecting home or work locations would certainly include travel cost between home and work. Thus two people living in close proximity and having a short commute time, may work together without influencing one another. In addition home location decisions can be driven by people wanting to be close to those that are demographically more like them leading to the selection of some neighborhoods more than others. Observed levels of homogeneity in neighborhood racial composition, and economic class suggest that a household's decisions are impacted by others even when those "others" may not be personally known. For example Massey et al. (1989) document considerable segregation along racial lines in the housing market in large urban areas of the US. Different rationales have been proposed for its existence including perceived social class difference, in-group preference (Clark, 1992), and prejudice (Massey and Denton, 1993; Bobo and Zubrinsky, 1996). Schelling (Schelling, 1978) demonstrates a model where small differences in tolerance can lead to segregated outcomes. Neighborhood segregation along income (Jargowsky et al., 1996) as well as age (Chevan, 1982) have also been documented. The analysis below seeks to investigate whether co-location among those that are spatially close to one another is significant even within such communities.

There is ample research in the field of sociology on the role played by social contacts in work finding. The economics literature on the other hand had widely adopted a search theoretic approach to the job search problem (see Devine and Kiefer, 1993 for a review). Pointing out the role social contacts play in job finding, Granovetter (1974) explains "job finding behavior is more than a rational economic process - it is heavily embedded in other social processes that closely constrain and determine its course and results." In his study, Granovetter had found that most jobs at least in the professional, management and technical fields were found via personal contacts (56.8\%). Granovetter $(1973,1974)$ work also identified which contacts were important in relaying information to find a job. He found that weak social ties, rather than strong ones with close family members and friends, were instrumental in relaying information that makes job finding possible. The rationale is that these individuals tend to move around in different circles from the job searcher and therefore are more likely to have information that the job seeker and their close contacts do not. People that have strong ties to the searcher are more likely to know the same information as the searcher, and therefore are not very useful in relaying new information (in contrast, in China, Bian 
(1997) finds strong ties to be more effective in getting jobs where influence was essential). The existence of a considerable number of (weak) social ties at the neighborhood level is shown in (Wellman, 1996). Here we ask whether these contacts are instrumental in work finding.

One of the biggest challenges in teasing out such relationship based outcomes at a large scale is the availability of data. In the next section we will discuss the data and methods we use to investigate the home and work relationships. That will be followed by an analysis of co-location patterns in eight areas in the Minneapolis-St. Paul metropolitan region. Finally, we investigate the relationship between comparatively high levels of co-location and different block level demographic variables and some conclusions are presented.

\section{Data}

The data for this study comes from the Longitudinal Employer-Household Dynamics (LEHD) program of the US Census Bureau which compiles data from federal and state sources U.S. Census Bureau, 2009. Here we use the prototype OD matrix for 2002 for the state of Minnesota. The OD data requires the linkage of residence and worker data that are gathered from the federal and state levels respectively. Description of the OD data is give in Marc Roemer, 2004 and much of the description of the data below borrows from this description.

Residence location data for the LEHD is gathered from the Census Bureau's Statistical Administrative Records System (STARS) database. Stars includes data compiled from records including federal tax forms, medicare, HUD public assistance, selective service registration and so on. The data is geocoded and locations for about $10 \%$ of the workers that are not geocoded are imputed.

Data on the work locations comes from States. In this case the ES-202 report from State of Minnesota's Covered Employment and Wages program is used. The data is reported by employers whose employment is covered under Unemployment Insurance. The Minnesota data is estimated to cover 97\% of non-farm employment in Minnesota Minnesota, 2009.

The worker-employer link in the LEHD data is derived from Unemployment Insurance wage reports supplied by the State of Minnesota. Part of the strength of the Minnesota data is that work locations are reported at the establishment level within an employer allowing more accurate $\mathrm{O}-\mathrm{D}$ matching. Some states reports may show the head office but not identify the establishment at which a particular worker is employed requiring additional models to assign work locations.

For confidentiality, the LEHD reported OD trips only if the origin had at least five workers living in it, and if the trip makers had three or more destination blocks. Later versions of the LEHD have used other methods to preserve confidentiality.

We have limited our analysis to origins and destinations within the seven-county metropolitan area of the Twin Cities (Minneapolis-St. Paul). ${ }^{1}$ In the following sections we will investigate the extent of co-location at home and work between residents of several blocks and study how the degree of co-location varies across the demographic make up of the census blocks. $^{2}$

For the first part of the analysis eight areas, each constituting several census blocks are selected. Four of these were selected in the city of Edina and four in the city of Brooklyn Park. The number of census blocks in the selected areas ranged between 11 and 23. Each group is selected to have approximately 500 workers. The second part of the analysis will look at how co-location at work is distributed across the metropolitan area. The extent of co-location is analyzed against the demographic makeup of the census blocks utilizing demographic data from the US decennial census. The analysis will include all metro area census blocks where the origin had at least two workers originating from it to any metro area work block.

\section{Methodology}

We are interested in testing (i) whether work census-block sharing among people who share a home census-block is higher than what we would expected by travel and land use considerations and (ii) which census-blocks exhibit relatively higher home-work sharing. The first of these questions would indicate the presence of a coordination mechanisms among people in their choice of home and work locations. This would support the neighborhood and work place information flow hypothesis we have presented above. The second part of the analysis would explain what type of neighborhoods exhibit it strongly relative to others.

The first part of the analysis will borrow methods from Social Network Analysis. We measure the extent of home-work co-location by building adjacency matrices for residents of the areas under consideration at home and at work. A network adjacency matrix is a matrix representation of the relations between the members of a network. Because of the level of detail in the LEHD data set, we define a relationship as sharing a census block for residence or sharing census block as a work destination. The home and work relationships are depicted in two adjacency matrices, one for the residence end $(H)$ and one for the work place $(W)$. For a selected area that has $N$ workers as residents, the adjacency matrices for the $H$ and $W$ matrices will be $N \times N$ in dimension, where each row and column represents one individual. Cell $[i, j]$ of the $H$ matrix will have a value 1 if person $i$ and person $j$ share a home location or 0 otherwise. The same element in the $W$ matrix will have a value of 1 if $i$ and $j$

\footnotetext{
1 The seven counties are Anoka, Carver, Dakota, Hennepin, Ramsey, Scott, and Washington counties.

${ }^{2}$ A census block is the smallest geographic area for which the Census Bureau presents data. Blocks are grouped together to form block groups.
} 
work together and 0 otherwise. Both $H$ and $W$ are symmetric matrices. The diagonal elements are ignored as they do not have any meaning. For an area that has $N$ residents there are $N(N-1) / 2$ possible relationships.

Having defined the home and work matrices, our study focuses on the relationship between these matrices. Specifically we ask what the degree of association between these two matrices is when both matrices are defined over the same subset of individuals. We are also interested in running statistical tests on the level of association between the matrices. We employ three measures to measure the level of association between the home and work matrices. The measures of association are Pearson correlation, simple matching and Jaccard coefficient. Simple matching and the Jaccard coefficients are calculated as follows:

Simple matching: The simple matching coefficient measures to what extent the two matrices at the home and work block level are similar entry by entry. There are $n(n-1) / 2$ relationships defined in each matrix. This measure tells us what proportion of these relationships are equal to one another in the home and work adjacency matrices. For instance a 0.6 value means $60 \%$ of the relationships defined at home and work are the same (60\% of relationships are absent or present at both). The measure is calculated as follows:

$$
M=\frac{C}{C+D}
$$

where $C$ is the count of matched pairs (0-0 or 1-1) and $D$ is the count of un-matched pairs $(0-1$ or $1-0)$ between the $H$ and $W$ matrices.

Jaccard coefficient: The Jaccard coefficient looks at what proportion of possible ties are matched between the home and work location relationships. This measure excludes all values that are matched $0 \mathrm{~s}$ in the home and work adjacency matrices.

$$
J=\frac{C_{11}}{C_{11}+D}
$$

where $C_{11}$ is the count of matched $1-1$ pairs and $D$ is the count of unmatched pairs (0-1 or $\left.1-0\right)$ between the $H$ and $W$ matrices.

One of the challenges in applying statistical tests to relationship/network data is that observations are not independent of one another. Each entry of the matrix reports the presence/absence of a relationship between two actors, thus observations on relationships involving the same actor are not independent of one another. This makes hypotheses tests that assume independence between observations less useful. Social scientists have been dealing with such data in other contexts. One solution that has been used to address this problem is using quadratic assignment procedure (QAP) (Hubert and Schultz, 1976).

QAP is a non-parametric test that depends on repeated permutation of one of the network matrices and building a distribution of the test statistic of association under the null hypotheses. The permutation preserves the structure of the network while it relabels the vertices of the network. In our case for instance, if we were permuting the work matrix $W$, we would be exchanging relationships among the individuals in consideration, while maintaining the size of the clusters. From a trip distribution stand point, one can think of this as reassigning the destination blocks of the agents in the network while maintaining employment levels at each of the destination blocks. Fig. 1 illustrates what the process does in the context of our analysis. The relationships at the home location remain unchanged through out the procedure. Under successive permutations, the number of people employed at each of the destination blocks under consideration also remains unchanged. The difference is that at each iteration the employees that fill the positions are now pulled from different origin blocks. For example in Fig. 1 the destinations have 3, 3 , 3 and 1 employees respectively under the observed network as well as under permutations. However, different individuals are fill these positions under the permutations.

It is important to note that the QAP test, as well as the $H$ and $W$ matrices, do not explicitly control for origins and destinations. The method is generic enough to be applied to any type of network. The only information contained in the matrices is which groups of individuals are related and the structure of the network that develops (e.g. how many ties are there? how many cliques, etc.). Under permutation, the structure of the network is unaffected. For instance the total number of ties, the number cliques as well as the size of each clique does not change. The QAP test simply exchanges the relationships in the $W$ matrix and calculates the test statistic of interest against the $H$ matrix. After several iterations, the distribution of the test statistic under permutation is compared to the original test statistic. The percentage of statistic under permutation that are as great or greater than the original test statistic serves as the $p$-value of the test.

To answer whether work census block sharing is higher among people who share a home census-block, we start by selecting several areas and build the $H$ and $W$ relationship matrices for workers in living in the selected area. Our selection strategy is for each area to include a number of census blocks that are adjacent to one another and to analyze each group separately. As will be illustrated in the next section, there are certain advantages of selecting census blocks that are close to one another. First blocks that are close to one another tend to be more socio-demographically homogenous than a randomly selected set of blocks. Our intention is to illustrate that even among a demographically homogenous group, people who are closer to one another tend to work together than those slightly farther from them. Second distances to different land uses around them are relatively similar. Third, by selecting home blocks that are adjacent to one another we are more or less maintaining the overall origin-destination distance distribution. If one were to map the destination locations under permutation, the home to work distance of any one individual can change significantly from the original depending on whose position they are now assigned in the network. However, because the selected residential locations are very close to one another and the distance between homes is small, the overall distribution of distance is not affected significantly.

In permuting the adjacency matrix we start by generating a permutation vector. A simple way to think about this vector, and one which works with our context of reassigning destinations, is that its $k$ th element of the vector designates which 


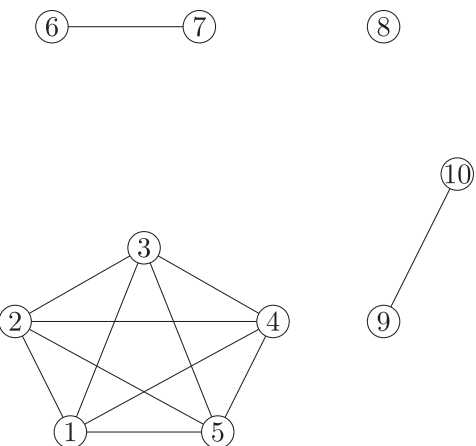

(a)Home relationships
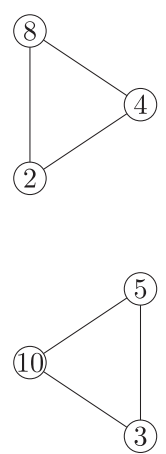

(c)Permuted work relationships 1
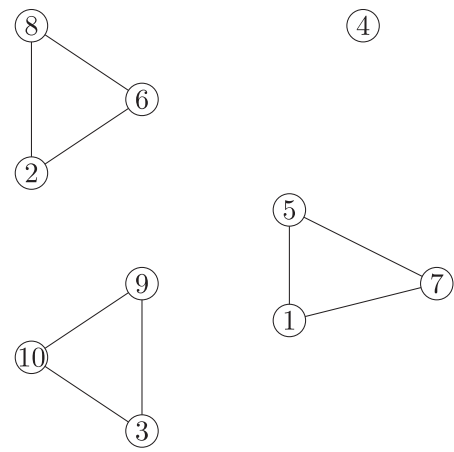

(b)Work relationships
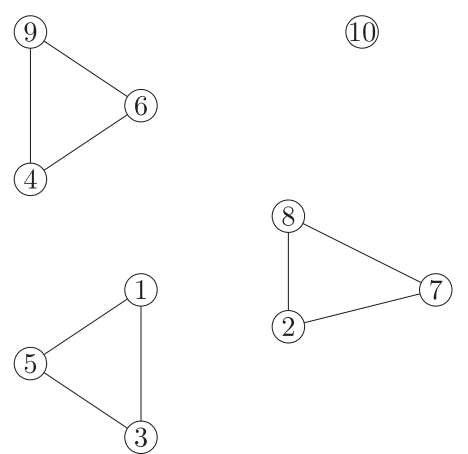

(d)Permuted work relationships 2

Fig. 1. Sample home and work networks.

position individual $k$ in the original matrix would take. Consider the simple adjacency matrix shown below which shows the work-co-location between five individuals along with the permutation vector and the adjacency matrix permuted format. Under permutation, the first person is now assigned the position previously held by person 2 and will have two ties. Because the other persons are also reassigned, the relationship is not necessarily with those person 2 had relationships with. If the home locations of persons 1-5 is the same, and they are now simply reassigned to the work locations of the element in the permutation vector, the overall distribution of distances will not change substantially.

$$
\left[\begin{array}{ccccc}
- & 0 & 0 & 1 & 0 \\
0 & - & 1 & 0 & 1 \\
0 & 1 & - & 0 & 0 \\
1 & 0 & 0 & - & 0 \\
0 & 1 & 0 & 0 & -
\end{array}\right]\left[\begin{array}{lllll}
2 & 1 & 5 & 3 & 4
\end{array}\right]\left[\begin{array}{ccccc}
- & 0 & 1 & 1 & 0 \\
0 & - & 0 & 0 & 1 \\
1 & 0 & - & 0 & 0 \\
1 & 0 & 0 & - & 0 \\
0 & 1 & 0 & 0 & -
\end{array}\right]
$$

The last point can be illustrated using one of the areas selected for the analysis in the next section. The area Brooklyn Park 2 in Table 1 includes 495 workers originating from 19 census blocks and destined to 263 census blocks. The resident's home coordinate is designated by the coordinates of the centroid of their home census block and their work coordinates are also designated by the centroid of the destination. Calculating the new home to work distance under 1000 different permutations and comparing the distance distribution to the actual distance distance distribution using a two sided t-test, no evidence of difference between the distributions was detected with $p$-values ranging from 0.865 to 0.970 .

Thus under the quadratic assignment procedure and the sampling strategy we have adopted (i) home locations remain unchanged (ii) employment levels at destinations remain unchanged and (iii) the overall distance distribution also remains relatively the same.

\section{Analysis}

\subsection{Measuring association between home and work co-location}

We perform the QAP analysis described above on eight areas in the cities of Edina and Brooklyn Park, Minnesota, part of the Twin Cities Metropolitan region. Four areas are randomly selected with in each city. Each of the selected areas had several blocks within it and approximately 500 workers destined to different blocks in the metropolitan area. Detailed results 
Table 1

Quadratic assignment procedure results.

\begin{tabular}{|c|c|c|c|c|c|c|}
\hline Group & Number of origin blocks & Number of destination & Number of workers & Association measure & Observed & $\%$ Greater ( $p$-value) \\
\hline \multirow[t]{3}{*}{ Edina 1} & 15 & 235 & 495 & $\mathrm{~J}$ & 0.03 & 0.00 \\
\hline & & & & $\mathrm{S}$ & 0.91 & 0.00 \\
\hline & & & & $\mathrm{C}$ & 0.08 & 0.00 \\
\hline \multirow[t]{3}{*}{ Edina 2} & 21 & 256 & 504 & $\mathrm{~J}$ & 0.03 & 0.00 \\
\hline & & & & $S$ & 0.93 & 0.00 \\
\hline & & & & $\mathrm{C}$ & 0.09 & 0.00 \\
\hline \multirow[t]{3}{*}{ Edina 3} & 11 & 223 & 481 & $\mathrm{~J}$ & 0.03 & 0.00 \\
\hline & & & & $S$ & 0.93 & 0.00 \\
\hline & & & & $\mathrm{C}$ & 0.05 & 0.00 \\
\hline \multirow[t]{3}{*}{ Edina 4} & 23 & 241 & 511 & $\mathrm{~J}$ & 0.03 & 0.00 \\
\hline & & & & $\mathrm{S}$ & 0.93 & 0.00 \\
\hline & & & & C & 0.10 & 0.00 \\
\hline Brooklyn & 19 & 263 & 495 & $\mathrm{~J}$ & 0.03 & 0.00 \\
\hline \multirow[t]{2}{*}{ Park 1} & & & & $\mathrm{~S}$ & 0.92 & 0.00 \\
\hline & & & & $\mathrm{C}$ & 0.10 & 0.00 \\
\hline Brooklyn & 22 & 263 & 494 & $\mathrm{~J}$ & 0.03 & 0.00 \\
\hline \multirow[t]{2}{*}{ Park 2} & & & & $\mathrm{~S}$ & 0.93 & 0.00 \\
\hline & & & & $\mathrm{C}$ & 0.12 & 0.00 \\
\hline Brooklyn & 13 & 307 & 606 & $\mathrm{~J}$ & 0.01 & 0.00 \\
\hline \multirow[t]{2}{*}{ Park 3} & & & & $\mathrm{~S}$ & 0.58 & 0.00 \\
\hline & & & & $\mathrm{C}$ & 0.03 & 0.00 \\
\hline Brooklyn & 17 & 251 & 447 & $\mathrm{~J}$ & 0.03 & 0.00 \\
\hline \multirow[t]{2}{*}{ Park 4} & & & & $S$ & 0.92 & 0.00 \\
\hline & & & & C & 0.11 & 0.00 \\
\hline
\end{tabular}

for one group of blocks in Edina will be presented and we will summarize the results for the remaining groups of census blocks in Table 1.

The first group of blocks in Edina (Edina 1) contains 15 census blocks from which 495 workers originate. The workers are destined to 235 census blocks for work. Under permutation, people are assigned new co-location relationships at the work location. The area is also demographically relatively homogeneous as compared to the city of Edina.

Table 1 shows the results of the QAP analysis using all three measures discussed above. In each case, while the magnitude of the measures are low, they exist to a degree that cannot be easily replicated by exchanging the work locations of the individuals. The last column in the table in the column serves as the measure of $p$-value of the test. In 1000 permutations, no measure of simple matching, Jaccard coefficient or correlation as large as that in the observed network was observed.

The results for the three other groups of blocks in Edina and the four groups of blocks in Brooklyn Park are also similar (see Table 1). The association measures under permutation are less than what is observed in the observed arrangement.

By selecting census blocks that are close to one another we have attempted to control for distance impacts as illustrated earlier. The permutation process itself ensures that the total number of persons working together is kept the same by retaining the structure of the network. Only the identities (and hence where they are attracted from) of the individuals that are working together is changed. The results suggest is that there are possible mechanisms that lead to co-location patterns that are significantly different from what are possible arrangements of employment.

A shortcoming of this type of analysis is of course that not all jobs are exchangeable. Individuals could in reality also be restricted in their choices of job location by the type of employment that is available. So the distribution on which the null hypothesis is based upon may not be tenable in reality. On the flip side, there are certainly many more locations in the metropolitan area where fitting employment may be found suggesting home-work co-location at the block level may have other mechanisms behind it. Another concern is that intra-household information sharing might also be at play in the extent of co-location that is observed.

It is instructive however to study the relationship between the degree of co-location and the socio-demographic characteristics of the each of the census blocks in the metropolitan area. For instance if locations that have more renters than homeowners show lower co-location, this would be inline with our hypothesis of social network influence. This is because there is little reason why intra-household effects would be different between renters and owners other things equal. However the degree to which people know their neighbors would be expected to be higher for home owners than for renters. The next section examines the relationship between aggregate socio-demographic characteristics of residential blocks based on the 2000 US census and the level of co-location each block exhibits calculated using the LEHD data.

\subsection{Home-work sharing across census blocks}

This section will look at how home-work co-location varies across the census blocks in the metropolitan area. Data for this portion of the analysis comes from the US Census Bureaus decennial national census. The variables in our model used below come from the $100 \%$ characteristics form that is asked of every person and housing unit in the country and released aggregated at the census block level. 
Table 2

Origin block characteristics.

\begin{tabular}{|c|c|c|c|}
\hline Variable & Description & Category & Percentage (\%) \\
\hline \multirow[t]{4}{*}{$R$} & Race & White & 95.80 \\
\hline & & Black & 2.50 \\
\hline & & Asian & 1.03 \\
\hline & & Other & 0.66 \\
\hline \multirow[t]{5}{*}{$A$} & Age & $<30$ & 21.42 \\
\hline & & $30-40$ & 48.42 \\
\hline & & $40-50$ & 23.18 \\
\hline & & $50-65$ & 5.75 \\
\hline & & $>65$ & 1.24 \\
\hline \multirow[t]{4}{*}{$H$} & Percentage of one person households & $>75 \%$ & 1.14 \\
\hline & & $50-75 \%$ & 4.47 \\
\hline & & $25-50 \%$ & 26.52 \\
\hline & & $<25 \%$ & 67.86 \\
\hline \multirow[t]{4}{*}{0} & Percentage of owner occupied dwellings & $>85 \%$ & 69.90 \\
\hline & & $50-85 \%$ & 16.23 \\
\hline & & $25-50 \%$ & 6.03 \\
\hline & & $<25 \%$ & 7.84 \\
\hline \multicolumn{2}{|c|}{ Total blocks used } & 32494 & \\
\hline
\end{tabular}

The unit of analysis for this portion of the study is the census block. As such the $H$ matrix for all blocks contains only $1 \mathrm{~s}$ and has no variation across blocks except for its size. To compare which census blocks have relatively higher incidence of people working together, we will use the density of the $W$ matrix for the residents of each block.

The density of a network measures what proportion of possible ties are realized. Its value can range between 0 and 1 . For a census block with $N$ workers, the density of the work relationship is the count of ties (1 s) in the upper triangle of the $W$ matrix divided by $n(n-1) / 2$. If everyone in the home block works at the same destination, the density of the $W$ network is equal to 1 . If on the other hand everyone in the census block goes to their own unique census block for work, then the density is 0 since no ties are present in the work matrix. Coincidentally, because the entries of the $H$ matrix are all ones, the simple matching, as well as the Jaccard coefficient calculated for a single block are also equal to the density of the work block.

A logit model is used to analyze the relationship between density and block characteristics. To control for the opportunities that are available from each residence area and the ease of accessing them, gravity based accessibility measures as estimated in El-Geneidy and Levinson, 2006 for the Twin Cities are used. The gravity based measures use the LEHD number of job reported for each of the blocks aggregated to the TAZ level and uses the reciprocal of the square of the travel time between TAZs for impedance (see El-Geneidy and Levinson, 2006 for more details). In addition to accessibility measures, racial composition as well as variables that control for demographic characteristics of each block are used. The proposed model is as follows:

$$
\rho=f\left(A_{b}, R, A, H, O, F\right)
$$

where $\rho$ is the tie density of the $W$ matrix, $A_{b}$ the gravity based accessibility measure of the TAZ that the block is in $(/ 1000), R$ the majority race in the block, $A$ the median age in the block, $H$ the percentage of one person households in the block, $O$ the percentage of owner occupied households in the block, and $F$ the average family size in a block.

The distribution of these area characteristics in the study area is given in Table 2. In fitting the model, we have controlled for the number of workers originating from the block. The density measure goes down at a much faster rate for each additional individual added to the network since one additional individual means $n-1$ possible ties in the network. Initial fitting of the model showed over dispersion as evidenced by the ratio of the deviance and the degrees of freedom. A scale was estimated by the square root of the the deviance divided by the degrees of freedom. The scale does not affect the estimates but rescales the covariance matrix. The final model is as shown in Table 3.

Blocks that have relatively higher accessibility measures exhibit higher incidences of working together. In part this may be due to the ease of job access that individuals in such blocks have close to them. As the number of opportunities in a particular block grows the possibility of working at that particular location for any given individuals also grows. After controlling for employment accessibility, interesting relationships between block level demographic variables and home-work co-location.

Although the majority of home blocks are predominantly White, a fraction of the blocks (about $4 \%$ ) have populations that are predominantly Black, Asian, or "Other" (which includes Native American, Hispanic, or mixed communities). Compared to blocks that are predominantly White, blocks where a majority of the population is Black, Asian, or Other have higher tie densities at the work location. Especially predominantly Asian blocks exhibit larger co-location patterns. Blocks with Black or Other racial groups as the majority also tend to have higher co-location than whites ( $p$-value $=0.05$ ).

The model also illustrates that blocks where multi-person households constitute more than $75 \%$ of households have higher work-place density than all other categories $(p$-value $=0.00)$. No significant difference was detected between the other categories and the base category of greater than $75 \%$ single-person households. The finding suggests that location decisions 
Table 3

Logit estimate of workplace tie density by census block.

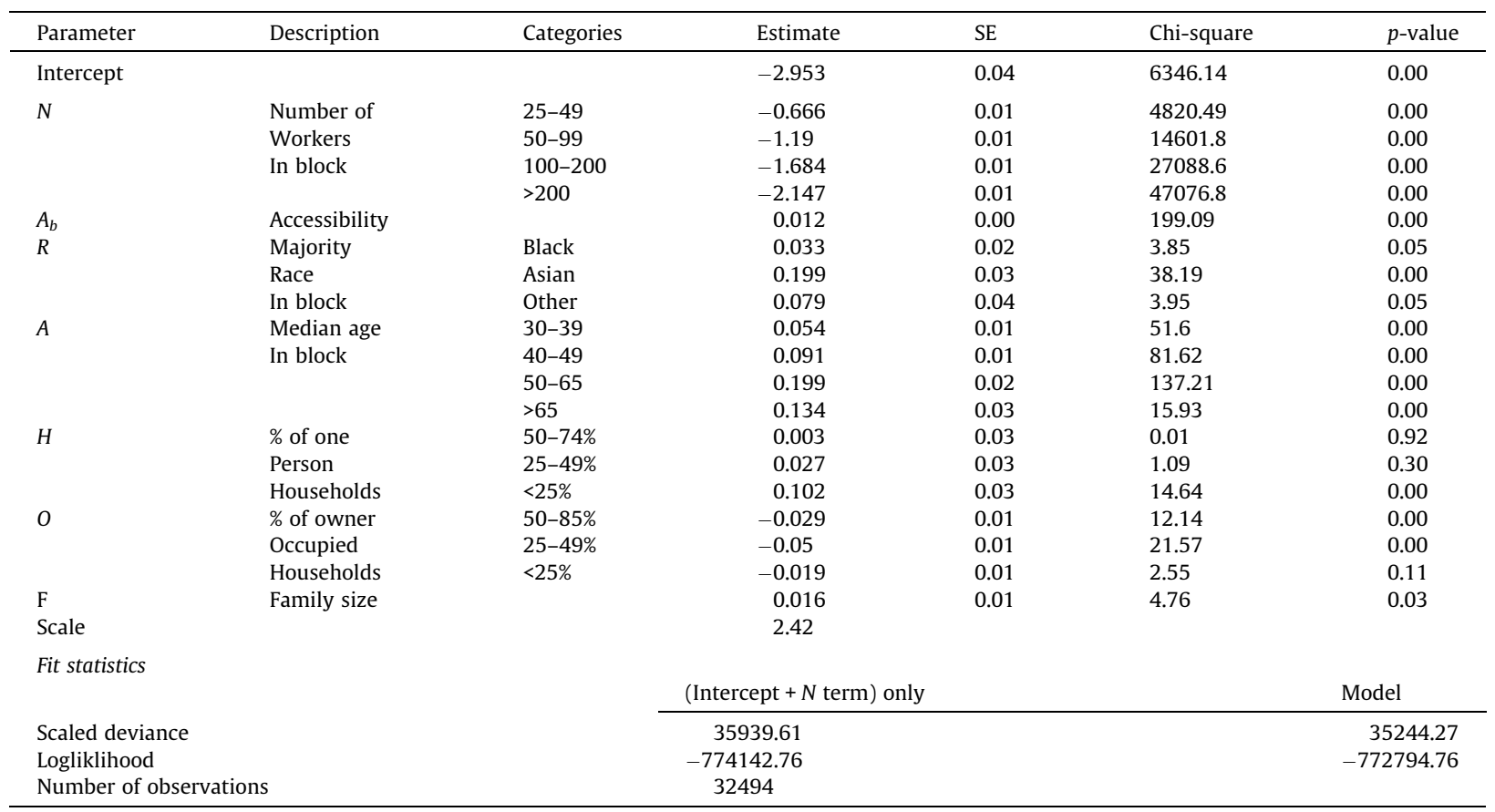

in multi-person households maybe significantly different that in single-person households. This may be because of withinhousehold effects, where people who live together also share a work location, or because individuals with multi-person households have better social networks within their neighborhoods and workplaces that lead to information transfer about jobs/houses.

As the median age in a block group increases, so does the possibility that people who live in that block also share a work block. The estimates indicate that odds of co-location are significantly higher for each age group as compared to the youngest of blocks. With age and experience individuals may better be able to exploit their networks at the neighborhood in getting employment, and may also want to locate closer to contacts that are much like them. They may also be ready to put more weight on neighborhood quality recommendations by others, and less likely to try out a new neighborhood on their own.

Finally, blocks that have a large number of owner-occupied dwellings have significantly higher incidence of people working together. As the proportion of owner-occupied dwellings goes down in a block, the probability that someone will work with another person in their residence block also goes down. This is possibly because home owners, who are likely to reside in their residences longer, form better social networks with those around them as opposed to renters who may relocate much more easily and see themselves as only temporarily in the area. Curiously blocks with less than $25 \%$ owner occupied households do not have a statistically significant lower incidence ( $p$-value $=0.11$ ). Blocks that have larger average family sizes also show larger incidence of home-work co-location.

Taken together, these results suggest that neighborhoods with older, predominantly multi-person, owner-occupied households tend to have a higher incidence of co-location at home and work than other neighborhoods. Co-location as measured by the workplace network density is higher in blocks that are occupied by mature and settled households.

Blocks that are predominantly Asian, Black or Other also display higher incidence of working together. In part this may be due to relatively segregated low-income job opportunities, but coupled with the QAP results earlier, it may also be indicative of communities having mechanisms in which co-location is maintained at a higher degree than predominantly White neighborhoods. Such patterns may in fact be pronounced in areas where new immigrants have settled where they are likely to depend on contacts to find a place of work.

\section{Conclusion}

This research hypothesizes that neighbors and co-workers play an important role in the choice of work and home by passing information about particular opportunities, homes or in general giving guidance on neighborhood quality. Location decisions that arise from these information flows could result in people living and working in closer proximity to one another with more frequency than would be expected otherwise.

We test this hypotheses using OD data at the census block level for the metropolitan area of Minneapolis-St. Paul. Quadratic assignment procedure is applied to eight areas in the Twin Cities that each contained several census blocks by defining 
home and work co-location as living in the same block and working in the same block. The results from the QAP analysis supports such a hypothesis by indicating that the observed association between home and work relationship matrices cannot be replicated by randomly exchanging the workplaces of the residents among themselves. That analysis is followed by a look at how network density as measured by the work place network for residents of each census block in the Twin Cities metro area varies with different demographic characteristics. The findings suggest that co-location is higher in blocks that are occupied by mature households (older, multi-person, home owner dominated blocks) while controlling for accessibility of the blocks to jobs.

Coupled with information about the spatial separation between work and home, understanding and explicitly including such mechanisms of location choice would theoretically improve our ability to predict the matching of origins and destinations. Such findings also give hope for exploring innovative solutions to encourage car-pooling among people who live and work close to one another, and in job-matching for people who are under or un-employed. Future work should utilize data that is more detailed and more specific on relationships than the LEHD origin-destination data can provide. Gathering relationship data of this scope is rather difficult and costly. However access to individual level variables could at least help further exploration by redefining relationships not only by census blocks but by demographic classes as well.

\section{References}

Arentze, T., Timmermans, H., 2008. Social networks social interactions and activity-travel behavior: a framework for microsimulation. Environment and Planning B: Planning and Design 35 (6), 1012-1027.

BarNir, A., Smith, K., 2002. Interfirm alliances in the small business: the role of social networks. Journal of Small Business Management.

Bian, Y., 1997. Bringing strong ties back in: indirect ties network bridges and job searches in China. American Sociological Review, 366-385.

Bobo, Lawrence, Zubrinsky, Camille L., 1996. Attitudes on residential integration: perceived status differences mere in-group preference or racial prejudice? Social Forces 74 (3), 883-909.

Calvó-Armengol, A., Zenou, Y., 2003. Does crime affect unemployment? The role of social networks. Annales d'Économie et de Statistique.

Carrasco, J.A., Miller, E.J., Wellman, B., 2006. Spatial and social networks: the case of travel for social activities. In: 11th International Conference on Travel Behaviour Research, Tokyo, Japan, August 2006, pp. 16-20

Cattell, V., people, Poor, places, poor, 2001. and poor health: the mediating role of social networks and social capital. Social Science \& Medicine.

Chevan, Albert, 1982. Age, housing choice and neighborhood age structure. American Journal of Sociology 87, 1113-1149.

Clark, William A.V., 1992. Residential preferences and residential choices in a multiethnic context. Demography 29 (3), $451-466$

Devine, Theresa J., Kiefer, Nicholas M., 1993. The empirical status of job search theory. Labour Economics 1 (1), 3-24.

Easa, S.M., 1993. Urban trip distribution in practice. I: conventional analysis. Journal of Transportation Engineering 119 (6), $793-815$

Easa, Said M., 1993. Urban trip distribution in practice. II: quick response and special topics. Journal of Transportation Engineering 119 (6), $816-834$.

El-Geneidy A, Levinson, D. Access to destinations: development of accessibility measures. Report 1 in the series access to destinations study. Department of Civil Engineering, University of Minnesota, May 2006.

Feld, S.L., 1981. The focused organization of social ties. American Journal of Sociology, 1015-1035.

Fleury, J., 1993. An exploration of the role of social networks in cardiovascular risk reduction. Heart \& lung.

Fontaine, F., 2008. Why are similar workers paid differently? The role of social networks. Journal of Economic Dynamics and Control.

Granovetter, Mark S., 1973. The strength of weak ties. American Journal of Sociology 78 (6), 1360-1380.

Granovetter, Mark S., 1974. Getting a Job: A Study of Contacts and Careers. Harvard University Press, Cambridge, Mass.

Hackney, J.K., Axhausen, K.W., 2006. An agent model of social network and travel behavior interdependence. In: 11 th International Conference on Travel Behaviour Research, Kyoto, 2006.

Hill, M., 1991. The role of social networks in the care of young children. Growing Up in a Changing Society.

Hubert, L., Schultz, J., 1976. Quadratic assignment as a general data analysis strategy. British Journal of Mathematical and Statistical Psychology 29 (1), 190241.

Jargowsky, Paul A., 1996. Take the money and run: economic segregation in U.S. metropolitan areas. American Sociological Review 61 (6), $984-998$.

Lindsay, C., Greig, M., McQuaid, R., 2005. Alternative job search strategies in remote rural and peri-urban labour markets: The role of social networks. Sociologia Ruralis 45, 53-70. Jan.

Roemer, Marc, 2004. A prototype of an origin-destination matrix for ETA-Excensus mapping pilot. Letter accompanying the LEHD data, December 2004.

Massey, Douglas S., Denton, Nancy A., 1993. American Apartheid: Segregation and the Making of the Underclass. Harvard University Press, Cambridge, Mass.

Massey, Douglas S., Denton, Nancy A., metropolitan areas: Black, Hypersegregation in u.s., dimensions, hispanic segregation along five, 1989. Hypersegregation in U.S. metropolitan areas: black and hispanic segregation along five dimensions. Demography 26 (3), $373-391$.

Minnesota Department of Employment and Economic Development. Key Definitions. http://www.deed.state.mn.us/lmi/publications/churn/dynam03.htm (accessed July 2009).

Pescosolido, B., Levy, J., 2002. The role of social networks in health, illness, disease and healing: the accepting present, the forgotten past, and the dangerous potential for a complacent future. <emeraldinsight.com> (January).

Powell, D., 1979. Family-environment relations and early childrearing: the role of social networks and neighborhoods. Journal of Research and Development in Education.

Schelling, Thomas C., 1978. Micromotives and Macrobehavior, first ed.. Fels Lectures on Public Policy Analysis Norton, New York.

Stoessel, S., 2002. Investigating the role of social networks in language maintenance and shift. International Journal of the Sociology of Language.

US Census Bureau, 2009. Longitudinal employer-household dynamics. Questions and answers. <http://lehd.did.census.gov/> (accessed June 2009).

Voorhees, Alan M., 1956. A general theory of traffic movement. In: 1955 Proceedings, institute of traffic engineers, New Haven, Connecticut, 1956.

Wellman, Barry, 1996. Are personal communities local? A dumptarian reconsideration. Social Networks 18 (4), $347-354$. 